\title{
FORCE ANALYSIS OF INDIVIDUAL MUSCLES ACTING SIMULTANEOUSLY ON THE SHOULDER JOINT DURING ISOMETRIC ABDUCTION:
}

\author{
CARLO J. de LUCA and W'ILLIAM J. FORREST'+ \\ Department of Anatomy. Queen's University. Kingston, Ontario, Canada
}

\begin{abstract}
A technique was developed for calculatine the force gencrated by individual muscics that contribute to isometric abduction of the upper limb in the coronal plane with the humerus medially rotated. The instantaneous center of rotation was located and used to indicate the muscles that had a force component which contributed to the force of abduction. Mathematical relationships were obtained for the forces of the individual muscles as a function of their respective 'effective' or physiological cross-sectional arcas. The cffective cross-sectional arca was expressed as a function of the maximum thickness of the middle fibers of the deltoid muscle - a parameter that could be mcasured casily on an X-ray film. The static equilibrium cquation about the instantaneous center of rotation could be solved. Any of the forces involved in ahduction could be expressed as a function of the measured force of atduction. In the process of applying this technique, certain functions of the muscles involved in the abduction of the upper limb were clarified.
\end{abstract}

\section{INTRODUCTION}

IT HAS been common practice among electromyographers to obtain a relationship between the myoelectric signal recorded from a single muscle and the force output of an isometric or isotonic muscle action about an casily monitored joint (Licht, 1971; Basmajian, 1967). This technique does not allow direct comparison between the force output of a muscle and its myoelectric signal.

Ideally, force measurements should be made on a joint which performs an action that is controlled by a single, large and easily' accessible muscle. Since such a situation does not exist in the human body, there are two possible alternatives. The first is to attach a force sensor directly to the tendon of the muscle being studied. This method would involve a surgical procedure, thus limiting or ruling out its use. The second alternative, the one adopted for this study, consists of the following two steps:

(a) choose an easily observable joint motion that is performed by as few muscles as possible. (h) devise a technique for calculating the forcc output of an individual muscle when the force of the joint motion is measured.

The joint motion studied in this investigation was abduction of the upper limb in the coronal plane with the humerus medially rotated.

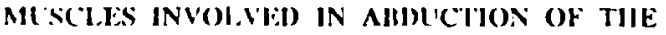 UPPER LIMB}

It is a ecnerally accepted fact that the chicf abductors of the urper limb are the deltoid and the supraspinatus muscles. There are four other muscles whose anatemical positions sugest possible direct involvement during atuluction. These are the long head of the biceps brachii, infraspinatus, teres minor and subscapularis muscles. Mycclectric investigations by Basmajian and Latif (1957) and physiological or 'natural method' investigations by Wright (1962) have proven that the long head of the bicers brachii is active during abduction only when the humens is rotated laterally; when the humerus is rotated medially and the forcarm is pronated. the long head of the bicers trachii is inactive.

The involvement of the three rotator-cuft muscles (infraspinatus, teres minor and subscapularis) mentioned above is not explicit. If these muscles truly do not contribute to the force output of the upper limb during abduction. the force of abduction will be provided only by the deltoid and supraspinatus muscles.

-Received 1 August 1972.

tDepartment of Physical Medicine and Rehabilitation. 
The supraspinatus muscle arises from the supraspinous fossa, passes directly over the shoulder joint and inserts on the summit of the greater tuberosity of the humerus. This muscle has a reasonably well defined origin and insertion. The deltoid muscle is a coarsely fasciculated multipennate muscle that can be divided into three anatomically and functionally distinct groups of fibers. The anterior fibers originate from the front of the lateril third of the clavicle, the middle fibers from the fitcral border of the acromion, and the posterior fibers from the inferior lip of the crest of the spine of the scapula. The fibers from the three parts converge and insert into the deltoid tuberosity, situated on the lateral aspect of approximately the middle of the shaft of the humerus.

The middle fibers of the deitoid muscle are located in a sagittal plane perpendicular to the plane of abduction. hence they are employed for abduction. The anterior and posterior fibers form curved surfaces which extend anteriorly and posteriorly from the plane of abduction. The anatomical function of these two groups of fibers is more complex. Their location suggests that during abduction they can perform two distinct functions: (a) oppose each other's anterior and posterior pull and thus help to stabilize the humerus in the plane of abduction, and (b) simultarieously act as abductors and/or adductors.

A myoelectric study by Yamshon and Bierman (1949) revealed that the anterior, posterior and middle fibers of the deltoid muscle are all active during abduction. Their observation was confirmed by the more detailed study of Scheving and Pauly (1959), who made the additional observation that the myoelectric signal from the middle fibers was greater than that from the anterior and posterior fibers, implying that the force output of the middle fibcrs was greater than that of the anterior or posterior fibers. Wright (1962) and Davies and Coupland in Gray's Anatomy (1969) both state that during abduction of the upper limb the anterior and posterior fibers of the deltoid muscle only serve to oppose each other's undesired actions, hence preventing side-sway.

\section{INSTANTANEOUS CENTER OF ROTATION OF THE UPPER LIMB}

The first step for finding the force contribution of individual muscles was to find the instantaneous center of rotation (ICR) of the upper limb during isometric abduction. In the shoulder joint, as in most joints, the center of rotation is not fixed in one location (Provins, 1965). However, the perturbation of the center of rotation is of no concern when dealing with isometric abduction; only the $\mathrm{CR}$ is of importance.

Five male subjects volunteered for the experiment. Their ages varied from 22 to 38 years, with an average age of 27.8 years. All subjects stated that they had not sustained injuries in their right shoulder area. An X-ray of the right shoulder area of each subject was taken with the upper limb medially rotated and abducted at an angle between 45.0 and $56.0^{\circ}$ (position 1). On the same film two additional exposures were made; one with the upper limb abducted at a slightly smaller angle (position 2) and the other with the upper limb abducted at a slightly larger angic (position 3). The complete film is shown in Fig. 1. The outline of the humerus in position 1 was traced on transparent paper. The transparent paper was rotated about a pivot point. By trial and crror. the correct pivot point which supcrimposed the outline of pesition 1 of the transparent paper onto the outline of position 2 of the film was found. This pivot point marked the ICR of positions 1 and 2 . The procedure was repeated for positions $I$ and 3. In the five X-ray films. the two ICR's did not coincide. indicating that the shoulder joint has a cen:er of rotation that varies with the angle of abduction and/or subject. The bisecting point of a straight line joining the two ICR's wats considered to be the ICK of the upper limb in position 1. The assumption that the ICR moves along a straight line is a first order approximation which has a small error if the displacement of positions 2 and 3 is small.

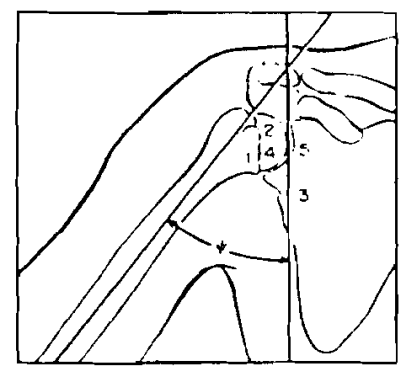

\begin{tabular}{|c|c|}
\hline Sute & $\begin{array}{l}\text { Angle of } \\
\text { oboution }\end{array}$ \\
\hline$\Delta T$ &. .541 \\
\hline fw & 4.526 \\
\hline$D E$ & 4.450 \\
\hline 4 & $\psi_{1}=483$ \\
\hline$\omega=$ & $\psi_{1}=560$ \\
\hline
\end{tabular}

Fig. 2. Location of the instantaneous center of rotation of the upper limb of the five subjects. The corresponding angles of abduction. $\psi$, between the center line of the limb and the sagittal plane are listed on the right of the figure.

In all cases, the ICR was situated medially and inferiorly to the greater and lesser tuberosities of the humerus, but not necessarily on the head of the humerus (see Fig. 2). The location of the infraspinatus, teres minor and subscapularis muscles is such that their resulting force components pass very close to the ICR. Therefore, these muscles will have a very small moment, if any, to contribute to the abduction of the upper limb. It appears that when the upper limb is abducted between the angles of 45.0 and $56.0^{\circ}$, the function of these muscles is to stabilize the humerus.

\section{FORCES CONTRIBUTING TO ISOMETRIC ABDUCTION}

The force output of the deltoid muscle can 


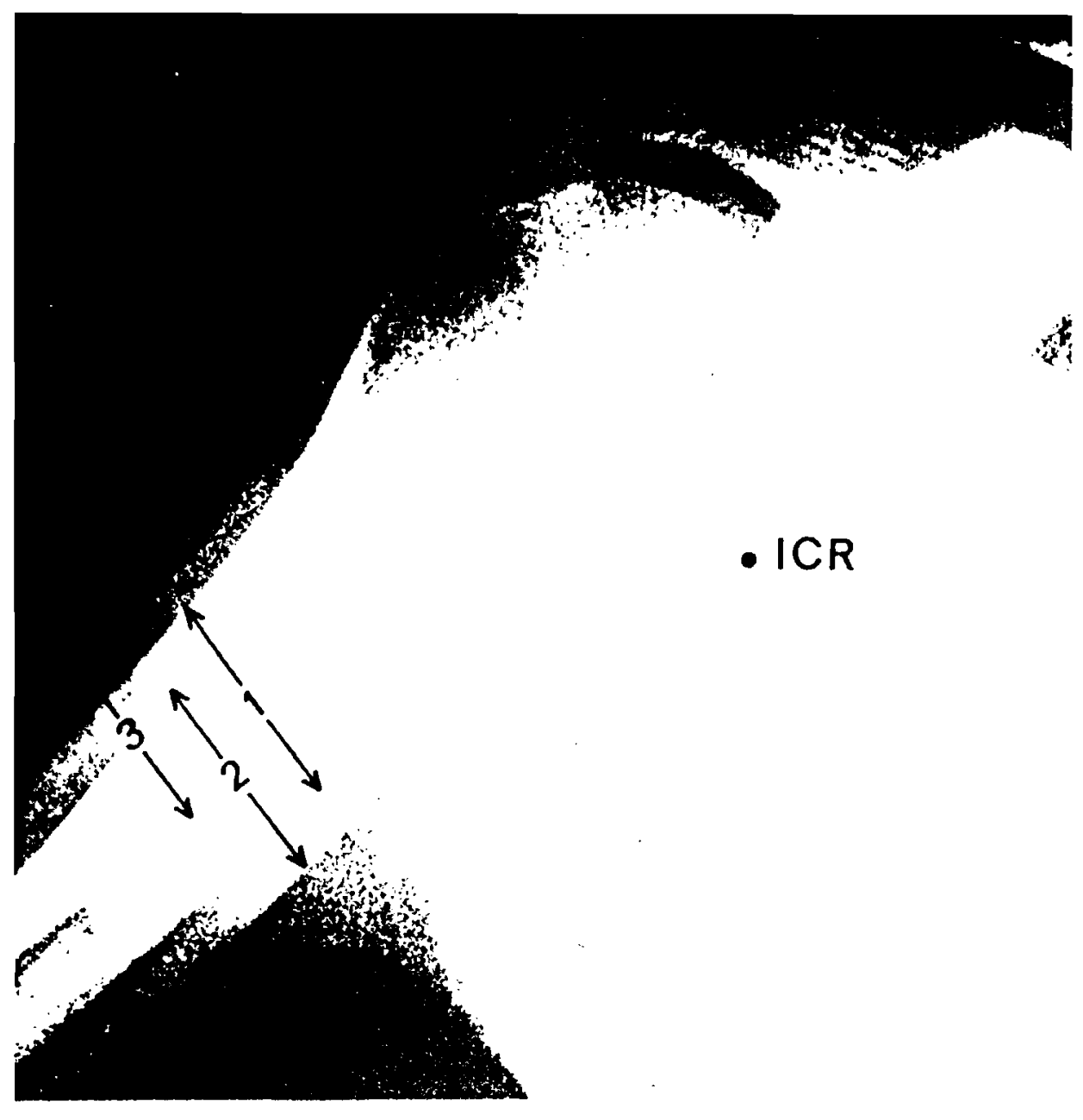

Fig. 1. X-ray film of the shoulder joint during abduction in the coronal plane. The middle position corresponds to the angle of abduction at which the measurements were taken. Note the location of the instantaneous center of rotation (ICR). 
be resolved into three separate force vectors, $f_{a}, f_{m}$ and $f_{p}$ as shown in Fig. 3a. The directions of the force vectors is obtained by connecting the apex of the insertion into the

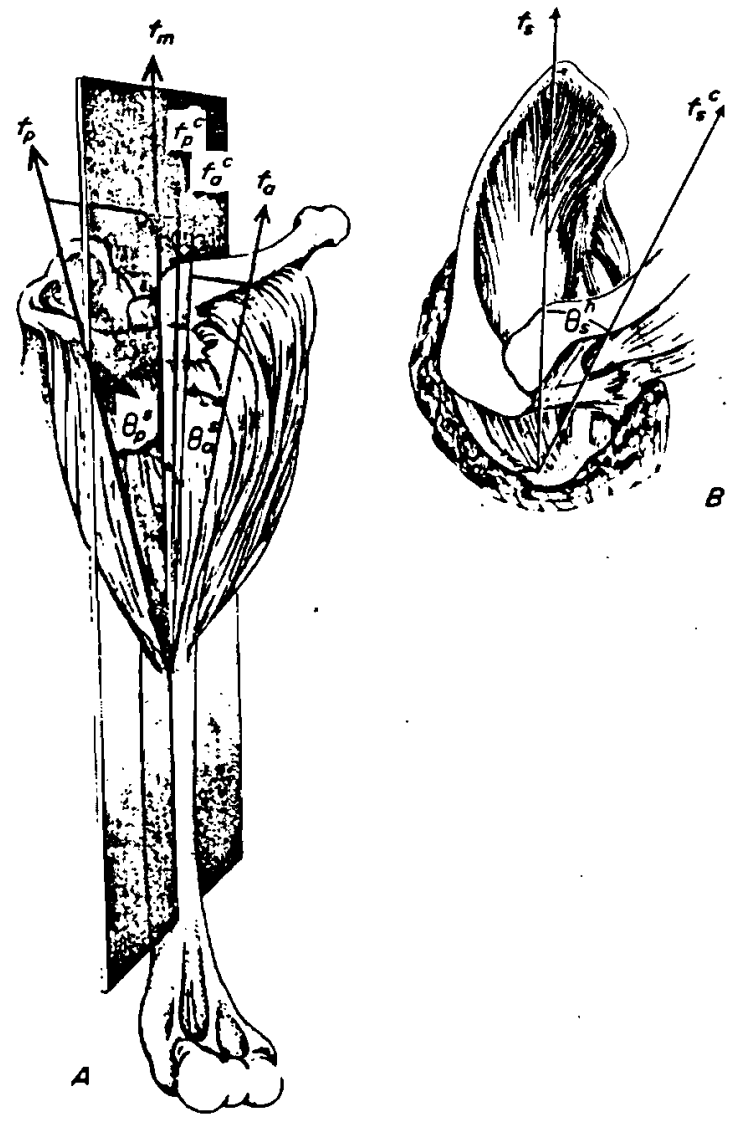

Fig. 3. (a) Force vectors of the anterior, middle and posterior fibers of the deltoid muscle and their projections on the coronal plane. (b) Force vector of the supraspinatus muscle and its projection on the coronal plane.

deltoid tuberosity with the middle of the origin of the respective muscle fibers. The magnitude of the vectors is unknown. The anatomical location of the middle fibers restricts the force vector $f_{m}$ to the coronal plane. The projection of the force vectors $f_{a}$ and $f_{p}$ on the coronal plane represents the force contribution of the corresponding fibers. The projections are obtained by the following relations:

$$
f_{a}^{c}=f_{a} \cos \theta_{a}^{s} \quad f_{p}^{c}=f_{p} \cos \theta_{p}^{s}
$$

where $\theta_{a}^{s}$ and $\theta_{p}^{*}$ are the angles on the sagittal plane between $f_{a}, f_{p}$ and the coronal plane. On the coronal plane, the angles between $f_{a}, f_{p}$ and the sagittal plane are denoted as $\theta_{a}^{c}$ and $\theta_{p}^{c}$. The force vector of the supraspinatus muscle, $f_{s}$, can be formed on the horizontal plane by connecting the middle of the greater tuberosity of the head of the humerus to the intersection of the spine and the medial border of the scapula as shown in Fig. 3b. The projection of $f_{z}$ on the coronal plane is represented by $f_{s}^{c}$ and can be calculated by the equation:

$$
f_{s}^{c}=f_{s} \cos \theta_{s}^{h}
$$

where $\theta_{s}^{h}$ is the angle on the horizontal plane between $f$, and the coronal plane. All the landmarks necessary for forming the force vectors can be easily palpated on the surface of the shoulder; hence, all the required angles can be measured with a goniometer.

Figure 4 represents a coronal-plane free-

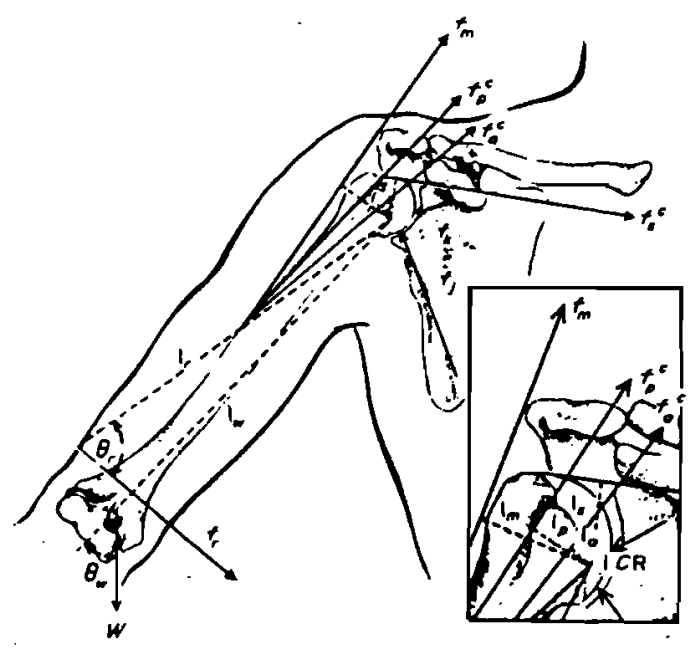

Fig. 4. Free-body diagram of the forces contributing to the isometric abduction of the upper limb.

body diagram of all the forces contributing to the isometric abduction of the upper limb against resistance. The symbols in the drawing have the following significance:
$f_{m}, f_{v}^{c}, f_{a}^{c}, f_{s}^{c}$ The forces of the deltoid and supraspinatus muscles projected onto the coronal plane.


$1_{m}, 1_{p}, 1_{a}, 1_{s}$ The perpendicular distance from the corresponding forces to the ICR.

$f_{r}$ The measured force of abduction.

$I_{r}, \theta_{r}$ The distance and angle which locate the position of $f_{r}$ with respect to the ICR.

$W$ The weight of the upper limb represented at the center of gravity of the upper limb.

$l_{w}, \theta_{w}$ The distance and angle which locate the position of the center of gravity of the upper limb with respect to the ICR.

$f_{j}, f_{k}$ The forces transmitted across the glenohumeral joint.

Values for the weight of the upper limb as a fraction of the body weight can be found in the literature. Dempster (1955) measured eight adult male cadavers ranging from middle to old age. He concluded that the right upper limb weighed 4.9 per cent of the total body weight and the left upper limb weighed 4.8 per cent of the total body weight. Bernstein (1967) measured 152 living subjects, males and females from 10 to 75 years of age (ratio of male to female not given). He found that for males the upper limb weighed $(5 \cdot 18 \pm 1 \cdot 13)$ per cent of the total body weight, whereas for females, the value was $(4.97 \pm 1.34)$ per cent. In the present study the subjects were righthanded males 22 to 38 years old. Due to the variation of the results of the above investigators; in this study, the weight of the right upper limb was arbitrarily chosen to be 5.0 per cent of the total body weight.

According to Dempster (1955) the center of gravity of the upper limb is located at 51.2 per cent of the distance from the glenohumeral axis to the ulnar styloid. This location corresponds to the center of the olecranon fossa. Braune and Fischer (1890) found that the centers of gravity of the limbs as well as other segments of the body are aligned on the coronal plane when a living body is in the normal standing position.
The direction of the forces transmitted across the glenohumeral joint can be found by observing the architecture of the scapula. The layer of bone forming the subscapular and infraspinous fossae is very thin. Two stout ridges of bone emanate from the glenoid fossa. These are the lateral border of the scapula and the intersection of the spine with the body of the scapula. These are the only two possible directions in which the bulk of the force can disperse when transmitted to the glenoid fossa. The force $f_{k}$ subsequently diverges to the coracoid process, the acromion and the spine of the scapula.

\section{MEASUREMENTS AND OBSERVATIONS ON THE SHOULDER JOINT}

The measurements in Fig. 4 were obtained by the following procedure. A soft-tissue coronal-plane X-ray of the upper limb and shoulder girdle was taken for each subject. The upper limb was abducted at the same angle used to obtain the ICR. The X-ray clearly outlined the deltoid muscle as well as the bone structure. The forces on the coronal planes of Figs. 3a and $b$ can be directly transposed onto the X-ray film. The point of action and the direction of $f_{r}$ and $W$ are known. The previously determined ICR (Figs. 1 and 2) was marked in the appropriate location. The pertinent measurements for each subject are listed in Table 1.

The value of $l_{a}$ and $l_{p}$ can be positive or negative. The distance is considered to be positive if the force vector is above the ICR and negative if it is below the ICR. Therefore, the anterior and posterior fibers of the deltoid muscle can function as abductors and/or adductors. For isometric abduction ranging from 45.0 to $56.0^{\circ}$, the anterior fibers act primarily as abductors and the posterior fibers primarily as adductors. Table 1 indicates that various abduction-adduction combinations are possible. Their relative function in an individual is related to the musculature arrangement about the shoulder joint and possibly the angle of abduction. 
Table 1. Surface and $X$-ray meastrements for the subjects

\begin{tabular}{|c|c|c|c|c|c|}
\hline \multicolumn{6}{|c|}{ Subjects } \\
\hline & LT & RW & DB & Ww & WF \\
\hline 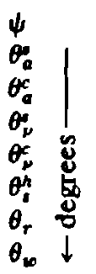 & $\begin{array}{l}54 \cdot 1 \\
16 \cdot 0 \\
18 \cdot 9 \\
22 \cdot 7 \\
26 \cdot 3 \\
49 \cdot 1 \\
85 \cdot 5 \\
43 \cdot 4\end{array}$ & $\begin{array}{l}52 \cdot 6 \\
11 \cdot 2 \\
16 \cdot 7 \\
16 \cdot 6 \\
21 \cdot 6 \\
49 \cdot 5 \\
67 \cdot 0 \\
47 \cdot 2\end{array}$ & $\begin{array}{l}45 \cdot 0 \\
17 \cdot 2 \\
17 \cdot 0 \\
14 \cdot 9 \\
17 \cdot 0 \\
49 \cdot 3 \\
79 \cdot 5 \\
51 \cdot 0\end{array}$ & $\begin{array}{l}48.3 \\
13.8 \\
14 \cdot 1 \\
32.0 \\
24.3 \\
49.0 \\
72.5 \\
43.6\end{array}$ & $\begin{array}{l}55 \cdot 0 \\
17 \cdot 1 \\
14.6 \\
22.2 \\
23.5 \\
47.8 \\
66.0 \\
36.0\end{array}$ \\
\hline $\begin{array}{ll}l_{m} & \uparrow \\
l_{a} & \mid \\
l_{p} & E \\
l_{s} & \delta \\
l_{r} & \\
l_{w} & \downarrow\end{array}$ & $\begin{array}{r}4.9 \\
0.2 \\
-2.1 \\
6.1 \\
19.9 \\
23.6\end{array}$ & $\begin{array}{r}5.3 \\
-1.2 \\
-3.1 \\
3.0 \\
24.3 \\
30.6\end{array}$ & $\begin{array}{r}8.7 \\
4.6 \\
4.6 \\
6 \cdot 3 \\
26.2 \\
30.8\end{array}$ & $\begin{array}{r}5.6 \\
1.3 \\
-1.8 \\
5.4 \\
24.6 \\
26.8\end{array}$ & $\begin{array}{r}8.3 \\
2.5 \\
-2.0 \\
3.4 \\
27.8 \\
29.4\end{array}$ \\
\hline$W(\mathrm{~kg})$ & 3.7 & $3 \cdot 7$ & 3.6 & 3.7 & $3 \cdot 8$ \\
\hline
\end{tabular}

RELATIONSHIP BETWEEN THE FORCES OF THE DELTOID AND SUPRASPINATUS MUSCLES

The diagram in Fig. 4 contains four unknown quantities; the magnitudes of $f_{a}, f_{m}, f_{p}$ and $f_{s}$.

A relationship between $f_{m}, f_{a}, f_{p}$ and $f_{s}$ may be obtained by calculating the absolute force of the individual groups of muscle fibers. The absolute force is defined as the maximum contractile force elicited by $1 \mathrm{~cm}^{2}$ of the effective or physiological cross-sectional area of - the muscle during a voluntary isometric contraction at approximately the resting length of the muscle. The effective cross-section is the area of a section perpendicular to all the fibers in a muscle. Two of the earliest investigators, Johnson (1903) and Fick (1910) found that human skeletal muscles exert an absolute force of 6 to $10 \mathrm{~kg} / \mathrm{cm}^{2}$. Rechlinghausen (1920) and Arkin (1938) found the absolute force of a muscle to be $3.6 \mathrm{~kg} / \mathrm{cm}^{2}$. Presumably, the foregoing investigators used male subjects. In a more detailed study, Morris (1948) found that the absolute force of a muscle was $9.2 \mathrm{~kg} / \mathrm{cm}^{2}$ and $7 \cdot 1 \mathrm{~kg} / \mathrm{cm}^{2}$ for male and female subjects respectively. In a more recent study involving 245 male and female subjects, Ikai and Fukunaga (1968) found the absolute force to be $4.7 \mathrm{~kg} / \mathrm{cm}^{2}$ independent of age and sex. Their measurements were restricted to the flexor muscles of the arm. The obvious discrepancy in the value of the absolute force for human skeletal muscle obtained by the above investigators is not germane to the present study. It is sufficient to note that the investigators concluded that a linear relationship exists between the force and the effective cross-sectional area of a muscle.

In living subjects, Ikai and Fukunaga (1968) measured the anatomical cross-sectional area of skeletal muscles by using ultrasonic equipment. In the case of the deltoid muscle, particularly the middle fibers, the effective and anatomical areas are not the same. Therefore, the following procedure was used to determine the effective cross-sectional area. The supraspinatus and deltoid muscles from the same shoulder were removed from six embalmed male cadavers ranging from middle to old age. The muscles were cut at the point of maximum cross-sectional area, located in the region where the nerve and blood supply enters the muscle. Care was taken to ensure that the plane of the cut always remained as perpendicular as possible to the muscle fibers. The anterior, middle and posterior fibers of the deltoid muscle were separated. A thin foil of paper was placed over the cross-section of the muscle, covering the complete area. The area was outlined on the paper and was measured with a planimeter; in each case, the area to be measured was traversed ten times.

It was necessary to find a parameter that could be measured on the subjects and correlated with the effective cross-sectional area of the muscles measured on the cadavers. The maximum thickness of the middle fibers of the deltoid muscle was found to be such a parameter. Figure 5 shows graphs of the effective cross-sectional area plotted against the maximum thickness of the middle fibers of the deltoid muscles of the cadavers. A linear leastsquare regression analysis was performed on the data and the resulting linear equations are plotted in Fig. 5. 


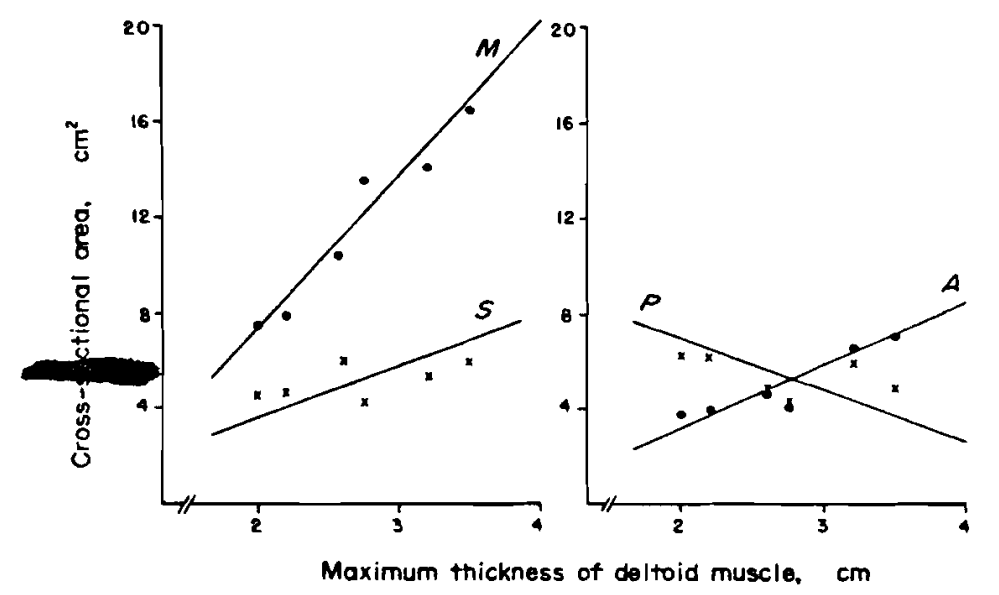

Fig. 5. The effective cross-sectional areas of the anterior, middle and posterior fibers of the deltoid and supraspinatus muscles plotted as a function of the maximum thickness of the deltoid muscle.

$$
\begin{array}{ll}
A_{\mathrm{a}}=-2 \cdot 3+2 \cdot 7 \rho & A_{\mathrm{p}}=11-2 \cdot 2 \rho \\
A_{m}=-5 \cdot 7+6 \cdot 5 \rho & A_{\mathrm{s}}=-0.64+2 \cdot 2 \rho
\end{array}
$$

where $A=$ the effective cross-sectional area of the muscle in $\mathrm{cm}^{2}$,

$\rho=$ the maximum thickness of the middle fibers of the deltoid muscle in $\mathrm{cm}$.

The corresponding correlation coefficients are

$$
\begin{array}{ll}
r_{a}=0.93 & r_{p}=-0.62 \\
r_{m}=0.97 & r_{d}=0.63 .
\end{array}
$$

The correlation coefficients of the anterior and middle fibers have a respective significance level of $p=0.007$ and $p=0.001$, implying that a highly significant linear relationship exists between the cross-sectional area of the anterior and middle fibers and the maximum thickness of the deltoid muscle. The significance level of the posterior fibers of the deltoid muscle and the supraspinatus muscle is $p>$ $0 \cdot 1$; this significance level does not establish a good linear relationship between the crosssectional areas of the posterior fibers of the deltoid and the supraspinatus muscles and the maximum thickness of the deltoid muscle. However, the latter two correlation coefficients do not exclude the possibility of a linear relationship. A better test for linearity could be made with more measurements, but at the time the measurements were made no additional cadavers were available.

It is interesting to note that the effective cross-sectional area of the posterior fibers is inversely related to that of the anterior fibers.

Equations (3) can be directly transformed to equations of force as a function of the maximum thickness of the middle fibers of the deltoid muscle.

$$
\begin{array}{ll}
f_{a}^{\prime}=\zeta(-2 \cdot 3+2 \cdot 7 \rho) & f_{p}^{\prime}=\zeta(11-2 \cdot 2 \rho) \\
f_{m}^{\prime}=\zeta(-5 \cdot 7+6 \cdot 5 \rho) & f_{d}^{\prime}=\zeta(-0.64+2 \cdot 2 \rho)
\end{array}
$$

where $f^{\prime}=$ the maximum force of the muscle in $\mathrm{kg}$,

$\boldsymbol{\zeta}=$ the constant of absolute force in $\mathrm{kg} / \mathrm{cm}^{2}$.

In live subjects, the maximum thickness of the middle fibers of the deltoid muscle was measured by taking coronal-plane soft-tissue $\mathrm{X}$-rays of the deltoid muscle. Three separate exposures were taken for each subject, one with the upper limb in the coronal plane, the other two with the upper limb slightly in front of and behind the coronal plane. The largest measurement obtained from the three X-ray 
Table 2. Maximum thickness (in $\mathrm{cm}$ ) of the middle fibers of the deltoid muscle of all subjects

\begin{tabular}{ccccc}
\hline LT & RW & DB & WW & WF \\
\hline 4.02 & 4.04 & 3.41 & 4.06 & 4.04 \\
\hline
\end{tabular}

films was considered to be the value of $\rho$. These values are listed in Table 2.

\section{RELATIVE FORCE CONTRIBUTION OF THE} SUPRASPINATUS AND DELTOID MUSCLES

It is necessary to know how the force output of each muscle contributing to the total force of abduction varies with respect to each other at various levels of abductory force.

During isometric contractions, the force of an 'unfatigued muscle' is a linear function of the integrated rectified myoelectric signal recorded from the muscle (Inman et al., 1952 and Lippold, 1952). On this basis, indwelling wire electrodes were inserted into the anterior, middle and posterior fibers of the deltoid muscle and into the supraspinatus muscle of three subjects. The other two subjects were not available. Each subject was seated in a chair specially equipped with a force gauge for measuring the force of isometric abduction of the upper limb (De Luca, 1972). The output of the force gauge was monitored on one channel of a dual-beam oscilloscope. The force gauge was calibrated to produce $4 \mathrm{~kg} / \mathrm{V}$. The other channel of the oscilloscope was connected to a ramp generator whose output was set to increase at a rate of $0.25 \mathrm{~V} / \mathrm{sec}$. The subject was asked to follow the ramp displayed on the oscilloscope as closely as possible by isometrically abducting the upper limb in the coronal plane. The required rate of force output was $1.00 \mathrm{~kg} / \mathrm{sec}$. The recorded myoelectric signals were rectified and integrated over successive periods of $0.25 \mathrm{sec}$. A special myolectric signal integrator designed and built in the EMG laboratory of the Anatomy Department at Queen's University was used.

It was found that for this particular experimental arrangement, the integrated rectified myoelectric signal was linear for a monitoredforce range of $0-12 \mathrm{~kg}$. Figure 6 illustrates the average normalized integrated rectified myoelectric signals of the anterior, middle and posterior fibers of the deltoid muscle and of the supraspinatus muscle with respect to the monitored force of abduction for three subjects. A linear least-square regression analysis revealed that the slopes of the four graphs in Fig. 6 have a maximum deviation of 3.7 per cent from their average value. This deviation is well within the estimated experimental accuracy of \pm 6 per cent. Hence, it can be concluded that for sub-maximal isometric abductions with a force output from $0-12 \mathrm{~kg}$,

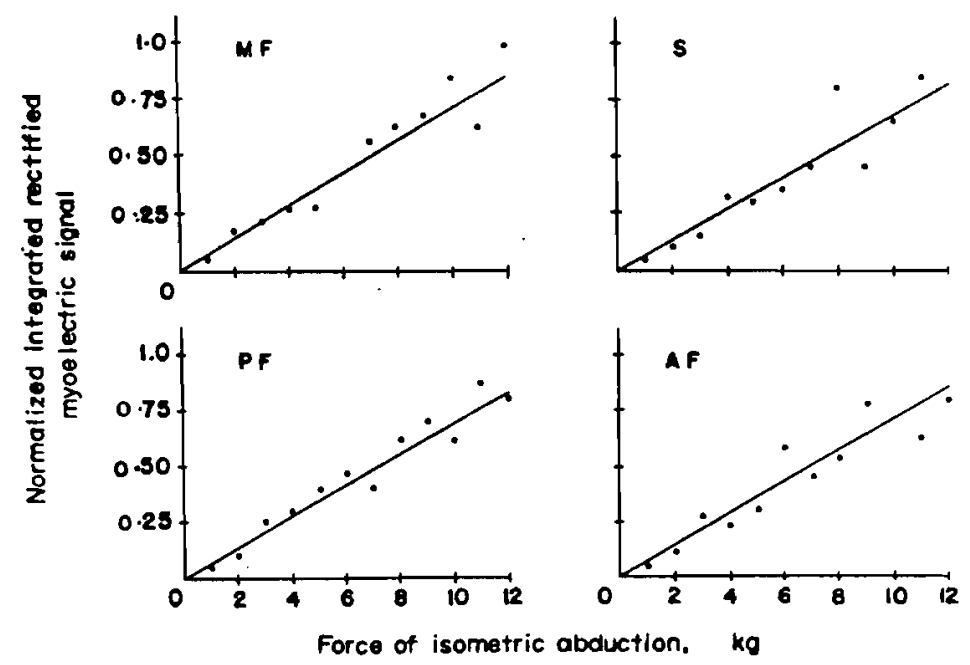

Fig. 6. The normalized integrated rectified myoelectric signal as a function of force of isometric abduction for the middle, posterior and anterior fibers of the deltoid muscle and the supraspinatus muscle. 
the ratio of the force contribution of the four groups of muscle fibers monitored remains constant. It is reasonable to assume, but difficult to prove, that the constant force-ratio between the groups of muscle fibers involved continues during stronger isometric contractions.

SYSTEM OF EQUATIONS FOR THE FORCES CONTRIBUTING TO ISOMETRIC ABDUCTION

Equations (4) may be rewritten for all levels of contraction

$$
\begin{array}{ll}
f_{a}=\zeta(-2 \cdot 3+2 \cdot 7 \rho) & f_{p}=\zeta(11-2 \cdot 2 \rho) \\
f_{m}=\zeta(-5 \cdot 7+6 \cdot 5 p) & f_{a}=\zeta(-0.64+2 \cdot 2 p) .
\end{array}
$$

Referring to Fig. 4, the static equilibrium equation about the ICR in the coronal plane can be written as

$$
\begin{aligned}
1_{s} f_{s}^{c} & +1_{m} f_{m}+1_{a} f_{a}^{c}+1_{p} f_{p}^{c}=1_{r} f_{r} \sin \theta_{r} \\
& +1_{w} W \sin \theta_{w .}
\end{aligned}
$$

Substituting equations ( 1 and 2 ) in equation (6) an expression with respect to the actual force vectors of the muscles can be obtained:

$$
\begin{gathered}
1_{s} f_{1} \cos \theta_{i}^{h}+l_{m} f_{m}+l_{a} f_{a} \cos \theta_{a}^{*}+l_{p} f_{p} \cos \theta_{p}^{*} \\
=1_{r} f_{r} \sin \theta_{r}+l_{w o} W \sin \theta_{w} .
\end{gathered}
$$

The force vectors $f_{j}$ and $f_{k}$ do not appear in the equations because their lines of action pass through the ICR. No other equilibrium equation can be written without introducing the force vectors of other muscles that are attached to the humerus.

Equations (5 and 7) form a system of equations of five equations and five unknowns, therefore, the forces $f_{a}, f_{m}, f_{p}$ and $f_{i}$ can be solved without knowing the value of the constant $\zeta$.

\section{SUMMARY AND CONCLUSION}

A method has been described whereby the individual force of a muscle or portion of a muscle can be measured while two or more muscles contribute to the force measured about a joint. The technique described consists of seven basic steps.

(1) X-rays of the joint studied were taken to determine the location of the instantaneous center of rotation (ICR).

(2) A soft-tissue $X$-ray was taken, showing the origin and insertions of the muscles involved in isometric abduction.

(3) The orientation of each muscle considered was described with respect to the plane of motion, in this case the coronal plane.

(4) The effective cross-sectional area of the muscles involved was measured on cadavers.

(5) The maximum thickness of one of the muscles contributing to the force of abduction was measured on a soft-tissue $X$-ray.

(6) The relative force contribution of the muscles involved was shown to be constant during sub-maximal isometric abductions.

(7) A set of equations describing the force relationship of all the muscles contributing to the force of abduction was developed and solved for the force of a particular muscle.

Steps four and five can be combined into one measurement made directly on the human subject if the fibers of the muscles affecting the particular joint have a simple geometrical arrangement.

As described in this paper, the method can only be used for isometric contractions. However, it may be extended to isotonic contractions if the location of the ICR can be expressed as a function of the angle of the joint and if the force-length relationship of the muscles is taken into consideration.

This study supports the belief that the main abductors of the upper limb are the middle fibers of the deltoid muscle and the supraspinatus muscle. The degree to which the other muscles surrounding the shoulder joint are involved in abduction is presently a subject of controversy. In this study, it was found that the infraspinatus, teres minor and the subscapularis muscles are mainly used to stabilize the upper limb during isometric abduction in the coronal plane. The anterior and posterior 
fibers of the deltoid muscle may act either as abductors or adductors while the upper limb is abducted, depending upon the location of their lines of action with respect to the ICR of the glenohumeral joint. For isometric abduction ranging from 45.0 to $56.0^{\circ}$, the anterior fibers act mainly as abductors and the posterior fibers mainly as adductors, but in some individuals both may simultaneously act as abductors or adductors. Their relative function in an individual is determined by the size and arrangement of the musculature about the shoulder joint and possibly the angle of abduction.

Measurements made on cadavers indicate that the physiological or effective crosssectional areas of the anterior and posterior fibers of the same deltoid muscle are inversely related. This striking result may partially explain the predominantly opposing function of the two groups of muscle fibers.

\section{REFERENCES}

Arkin, A. M. (1938) Absolute muscle power. The internal kinesiology of muscle. Research Seminar Notes, Dept. Orth. Surg., State Univ. of Iowa, Vol. 12D, 123.

Basmajian, J. V. and Latif, A. (1957) Integrated actions and functions of the chief flexors of the elbow: a detailed electromyographic analysis. J. Bone Jnt Surg. 39-A (5), 1106-1118.

Basmajian, J. V. (1967) Muscles Alive, 2nd Edn. Williams and Wilkins, Baltimore.

Bernstein, N. (1967) The Co-ordination and Regulation of Movements. Pergamon Press, Oxford.

Braune, W. and Fischer, O. (1890) Über den Schwerpunkt des Menschlichen Körpers mit Rücksicht auf die Ausriustung des Deutschen Infanteristen. Abh. d. math.-phys. Cl. d. k. Sächs. Gessellsch. d. Wiss., 15, 561-572.
Davies, D. V. and Coupland, R. E. (Editors) (1969) Gray's Anatomy, pp. 659-660. Longmans, Green and Co., London.

De Luca, C. J. (1972) A stochastic model for motor unit fring intervals and its applicability to myoelectric signals recorded during constant force isometric contractions in man. Ph.D. Thesis, Queen's University, Kingston, Ontario, Canada.

Dempster, W. T. (1955) Space requirements of the seated operator: geometrical, kinematic and mechanical aspects of the body with special reference to the limbs. WADC Technical Repor No. 55-159.

Fick, R. (1910) Handbuch der Anasomie des Menschen. Vol. 2, Gustav Fischer Verlas.

Ikai, M. and Fukunaga, T. (1968) Calculation of muscle strength per unit cross-sectional area of human muscle by means of ultrasonic measurements. Int. Z. Angewandie Physiol. 26, 26-32.

Inman, V. T., Ralston, H. J., Saunders, J. B. de C. M., Feinstein, B. and Wright, E. W., Jr. (1952) Relation of human electromyogram to muscular tension. EEG Clin. Neurophysiol. 4, 187-194.

Johnson, J. V. quoted by du Bois, R. (1903) Ergebnisse der Physiologie. Bd. 2, Heft 2,623.

Licht, S. (Editor) (1971) Elecirodiagnosis and Electromyography Licht, New Haven, Connecticut.

Lippold, O. C. J. (1952) The relation between integrated action potentials in a human muscle and its isometric tension. J. Physiol. 117, 492-499.

Morris, C. B. (1948) The measurements of the strength of muscle relative to the cross-section. Res. Quarr. Am. Assn. Health Phys. Ed. Recrn. 19, 295-303.

Provins, K. A. (1965) Ergonomics for industry. Men, machines and controls. Dept. Sc. Ind. Research, London.

Rechlinghausen, N. (1920) Gliedermechanik und Lahmungsprothesen. Springer, Bertin.

Scheving, L. E. and Pauly, J. E. (1959) An electromyographic study of some muscles acting on the upper extremity of man. Anat. Rec. 135, 239-245.

Wright, W. G. (1962) Muscle Function. Hafner, New York.

Yamshon, L. J. and Bierman, W. (1949) Kinesiologic electromyography -111 . The deltoid. Arch. Phys. Med. 30, 286-289. 